\title{
Global Dynamics of Systems Close to Hamiltonian Ones Under Nonconservative Quasi-periodic Perturbation
}

\author{
A. D. Morozov, K. E. Morozov
}

We study quasi-periodic nonconservative perturbations of two-dimensional Hamiltonian systems. We suppose that there exists a region $D$ filled with closed phase curves of the unperturbed system and consider the problem of global dynamics in $D$. The investigation includes examining the behavior of solutions both in $D$ (the existence of invariant tori, the finiteness of the set of splittable energy levels) and in a neighborhood of the unperturbed separatrix (splitting of the separatrix manifolds). The conditions for the existence of homoclinic solutions are stated. We illustrate the research with the Duffing - Van der Pole equation as an example.

Keywords: resonances, quasi-periodic, periodic, averaged system, phase curves, equilibrium states, limit cycles, separatrix manifolds

\section{Introduction}

Consider the system

$$
\begin{aligned}
& \dot{x}=\frac{\partial H(x, y)}{\partial y}+\varepsilon g\left(x, y, \omega_{1} t, \ldots, \omega_{m} t\right), \\
& \dot{y}=-\frac{\partial H(x, y)}{\partial x}+\varepsilon f\left(x, y, \omega_{1} t, \ldots, \omega_{m} t\right),
\end{aligned}
$$

Received April 14, 2019

Accepted June 20, 2019

This work has been partially supported by the Russian Foundation for Basic Research under grant no. 18-01-00306, by the Ministry of Education and Science of the Russian Federation (project no. 1.3287.2017/PCh) and by the Russian Science Foundation under grant no. 19-11-00280.

Albert D. Morozov

morozov@mm . unn.ru

Kirill E. Morozov

kirwamath@gmail.com

Lobachevsky State University of Nizhni Novgorod,

prosp. Gagarina 23, Nizhni Novgorod 603950, Russia 
where $\varepsilon$ is a small parameter, the Hamiltonian $H$ and the functions $g, f$ are sufficiently differentiable and uniformly bounded along with partial derivatives of order $\leqslant 2$ in a domain $G \subset \mathbb{R}$ or $G \subset R^{1} \times S^{1}$. Moreover, $g, f$ are assumed to be sufficiently smooth and quasi-periodic in $t$ uniformly with respect to $(x, y) \in G$ with incommensurable frequencies $\omega_{1}, \omega_{2}, \ldots, \omega_{m}$.

There are many works related to the topic. For instance, some specific examples of systems of form (1.1) were disscused in terms of complex dynamics in [1-3]. The Melnikov method is widely used in the literature as a criterion of chaos (for the Melnikov formula, see, e.g., [4-6]). A great number of papers refer to the existence of invariant tori in a weakly nonlinear case (see, e.g., [7-10]). There are also monographs concerning the averaging method (see [11-13]). In the present paper, we do not assume that (1.1) is quasi-linear and consider issues related to global dynamics along with local examination.

Suppose that the unperturbed system is nonlinear and has a region $D \subset G$ filled with closed phase curves $H(x, y)=h, h \in\left[h_{-}, h_{+}\right]$. The following condition is assumed to hold:

$$
\partial g / \partial x+\partial f / \partial y \not \equiv 0
$$

which implies that the system (1.1) is nonconservative. We also suppose that the system (1.1) with $\varepsilon=0$ has a separatrix loop of a saddle $\left(x_{s}, y_{s}\right)$. Note that $D$ contains neither a separatrix nor equilibria, nor their neighborhoods.

The global study of the system (1.1) involves examining the behavior of solutions both in $D$ and in a neighborhood of the unperturbed separatrix. In turn, there appear resonance energy levels in $D$. A closed phase curve $H(x, y)=h_{\text {res }}$ with the frequency $\omega\left(h_{\text {res }}\right)$ is called a resonance one if the frequencies $\omega\left(h_{r e s}\right), \omega_{1}, \ldots, \omega_{m}$ are commensurable.

Resonance levels are divided into passable, partly passable and impassable ones. If all the levels $H(x, y)=h_{\text {res }}$ are passable, then the qualitative behavior of (1.1) in $D$ is determined by the autonomous system

$$
\begin{aligned}
& \dot{x}=\frac{\partial H(x, y)}{\partial y}+\varepsilon g_{0}(x, y), \\
& \dot{y}=-\frac{\partial H(x, y)}{\partial x}+\varepsilon f_{0}(x, y),
\end{aligned}
$$

where

$$
\begin{aligned}
& g_{0}(x, y)=\frac{1}{(2 \pi)^{m}} \int_{0}^{2 \pi} \ldots \int_{0}^{2 \pi} g\left(x, y, \theta_{1}, \ldots, \theta_{m}\right) d \theta_{1} \ldots d \theta_{m}, \\
& f_{0}(x, y)=\frac{1}{(2 \pi)^{m}} \int_{0}^{2 \pi} \ldots \int_{0}^{2 \pi} f\left(x, y, \theta_{1}, \ldots, \theta_{m}\right) d \theta_{1} \ldots d \theta_{m} .
\end{aligned}
$$

It is assumed that the system (1.3) has only a finite number of limit cycles.

The paper [14] has outlined approaches to solving the problem of the global solutions behavior. Here we implement these approaches and illustrate them using the following equation as an example:

$$
\ddot{x}+\alpha x+x^{3}=\varepsilon f(x, y, t),
$$

where $\alpha= \pm 1, f=\left(p_{1}-x^{2}\right) \dot{x}+p_{2} \sin t \sin \nu t, p_{1}, p_{2}, \nu$ are parameters and $\nu$ is irrational.

In the case of periodic perturbations, the problem of the global solutions behavior for systems of type (1.1) was considered in [11] (see also [15]). The periodic analogue of (1.4) is studied in $[16,17]$. 


\section{Behavior of solutions in $D$}

In the action $I$-angle $\theta$ variables the system (1.1) takes the form

$$
\begin{aligned}
\dot{I} & =\varepsilon F_{1}\left(I, \theta, \theta_{1}, \ldots, \theta_{m}\right), \\
\dot{\theta} & =\omega(I)+\varepsilon F_{2}\left(I, \theta, \theta_{1}, \ldots, \theta_{m}\right), \\
\dot{\theta_{k}} & =\omega_{k}, \quad k=1,2, \ldots m,
\end{aligned}
$$

where

$$
\begin{aligned}
& F_{1}=f\left(x(I, \theta), y(I, \theta), \theta_{1}, \ldots, \theta_{n}\right) x_{\theta}^{\prime}-g\left(x(I, \theta), y(I, \theta), \theta_{1}, \ldots, \theta_{n}\right) y_{\theta}^{\prime}, \\
& F_{2}=-f\left(x(I, \theta), y(I, \theta), \theta_{1}, \ldots, \theta_{n}\right) x_{I}^{\prime}+g\left(x(I, \theta), y(I, \theta), \theta_{1}, \ldots, \theta_{n}\right) y_{I}^{\prime},
\end{aligned}
$$

the functions $x(I, \theta), y(I, \theta)$ define the change of variables, $\omega(I)$ is the natural frequency of the unperturbed system. Suppose that $\omega(I)$ is monotone on the interval $\left(I_{-}, I_{+}\right)$.

The phase space of the system $(2.1)$ is $\left[I_{-}\left(h_{-}\right), I_{+}\left(h_{+}\right)\right] \times T^{m+1}$, where $T^{m+1}$ is an $(m+$ +1 )-dimensional torus and $h_{-}, h_{+}$determine the boundaries of $D$. The functions $F_{1}, F_{2}$ are sufficiently smooth in $I, \theta, \theta_{1}, \ldots, \theta_{m}$. For $\varepsilon=0$, the $(m+2)$-dimensional phase space of the system $(2.1)$ is foliated by $(m+1)$-dimensional invariant tori $T^{m+1}$. For $\varepsilon \neq 0$, the tori can be destroyed by the nonconservativity of the perturbation and/or the presence of an integer combination of the frequencies $\omega, \omega_{1}, \ldots, \omega_{m}$ :

$$
n \omega(I)-(\mathbf{k}, \Omega)=0, \quad \mathbf{k}=\left(k_{1}, \ldots, k_{m}\right), \quad \Omega=\left(\omega_{1}, \ldots, \omega_{m}\right) .
$$

For given $\Omega$ and fixed $\mathbf{k}, n$, relation (2.3) can be viewed as an equation for $I$. If this equation has a real solution $I=I_{n \mathbf{k}}$ on the interval $\left[I_{-}, I_{+}\right]$, then the level $I=I_{n \mid b f k}$ (the closed phase curve $H(x, y)=h_{n \mathbf{k}}$ of the unperturbed system) will be called a resonance level.

Let us begin with consideration of the neighborhoods of individual resonance levels of energy.

\subsection{Neighborhood of a resonance level}

According to [14], the averaged system that describes the behavior of solutions in the neighborhood $U_{\mu}=\left\{(I, \theta): I_{n \mathbf{k}}-C \mu<I<I_{n \mathbf{k}}+C \mu, 0 \leqslant \theta<2 \pi, C=\right.$ const $\left.>0, \mu=\sqrt{\varepsilon}\right\}$ of resonance level $I=I_{n \mathbf{k}}\left(H(x, y)=h\left(I_{n \mathbf{k}}\right)\right)$ has the form

$$
\begin{aligned}
& u^{\prime}=A\left(v ; I_{n \mathbf{k}}\right)+\mu \sigma\left(v ; I_{n \mathbf{k}}\right) u, \\
& v^{\prime}=b_{1} u+\mu b_{2} u^{2},
\end{aligned}
$$

where the prime denotes the derivative with respect to the slow time $\tau=\mu t, b_{1}=\omega^{\prime}\left(I_{n \mathbf{k}}\right), b_{2}=$ $=\frac{1}{2} \omega^{\prime \prime}\left(I_{n \mathbf{k}}\right)$,

$$
\begin{gathered}
A\left(v ; I_{n \mathbf{k}}\right)=\frac{1}{(2 \pi n)^{m}} \int_{0}^{2 \pi n} \ldots \int_{0}^{2 \pi n} F_{1}\left(I_{n \mathbf{k}},\left(v+\left(\sum_{j=1}^{m} k_{j} \theta_{j}\right) / n\right), \theta_{1}, \ldots, \theta_{m}\right) d \theta_{1} d \theta_{2} \ldots d \theta_{m}, \\
\sigma\left(v ; I_{n \mathbf{k}}\right)=\frac{1}{(2 \pi n)^{m}} \int_{0}^{2 \pi n} \ldots \int_{0}^{2 \pi n}\left(g_{x}^{\prime}+f_{y}^{\prime}\right) d \theta_{1} \ldots d \theta_{m}
\end{gathered}
$$

where $x=x\left(I_{n \mathbf{k}}, v+\frac{1}{n} \sum_{j=1}^{m} k_{j} \theta_{j}\right), \quad y=y\left(I_{n \mathbf{k}}, v+\frac{1}{n} \sum_{j=1}^{m} k_{j} \theta_{j}\right)$. The functions $A\left(v, I_{n \mathbf{k}}\right)$ and $\sigma\left(v, I_{n \mathbf{k}}\right)$ are sufficiently smooth and periodic with the least period equal to $2 \pi / n$. The phase space of $(2.4)$ is a cylinder $(u, v \bmod 2 \pi / n)$. Simple equilibria of $(2.4)$ correspond to quasiperiodic solutions with $m$ frequencies in the initial system (i.e., $m$-dimensional invariant tori in the system (2.1)). The following theorem holds [14]. 
Theorem 1. Assume that the system (2.4) has a simple equilibrium $\left(v_{0}, 0\right)$ and $\sigma \neq 0$. Then the system (1.1) has a quasi-periodic solution $x(t), y(t)$ with frequencies $\omega_{1} / n, \ldots, \omega_{m} / n$ for sufficiently small $\varepsilon>0$. The solution is asymptotically stable if $b_{1} A^{\prime}\left(v_{0}\right)>0$ and $\sigma<0$ and unstable (saddle) if $b_{1} A^{\prime}\left(v_{0}\right)<0$. Accordingly, the system (2.1) has an m-dimensional stable (or saddle) invariant torus $T^{m}$.

Let us represent the function $A\left(v ; I_{n \mathbf{k}}\right)$ in the form: $A\left(v ; I_{n \mathbf{k}}\right)=B_{0}\left(I_{n \mathbf{k}}\right)+\widetilde{A}\left(v ; I_{n \mathbf{k}}\right)$, where $B_{0}$ is the mean of $A$. We shall say that $I=I_{n \mathbf{k}}$ determines a resonance energy level of the first type if $\max \left|\widetilde{A}\left(v ; I_{n \mathbf{k}}\right)\right|<\left|B_{0}\left(I_{n \mathbf{k}}\right)\right|, B_{0}\left(I_{n \mathbf{k}}\right) \neq 0$; a resonance level of the second type if $\max \left|\widetilde{A}\left(v ; I_{n \mathbf{k}}\right)\right|>\left|B_{0}\left(I_{n \mathbf{k}}\right)\right|, B_{0}\left(I_{n \mathbf{k}}\right) \neq 0$; a resonance level of the third type if $B_{0}\left(I_{n \mathbf{k}}\right)=$ $=0, \widetilde{A}\left(v, I_{n \mathbf{k}}\right) \not \equiv 0$. The qualitative behavior of (2.4) in the neighborhoods of levels of each type was established in [11]. If $\sigma$ is sign-preserving, then there exists $\varepsilon_{n \mathbf{k}}>0$ such that a first (second, third) type level determined by $I=I_{n \mathbf{k}}$ is passable (partly passable, impassable) if only $0<\varepsilon<\varepsilon_{n \mathbf{k}}$.

If the perturbed autonomous system (1.3) has a limit cycle in the neighborhood of the level $H(x, y)=h_{n \mathbf{k}}$, then this level is of the third type (i.e., impassable). Indeed, $B_{0}(I)$ is the Poincaré-Pontryagin generating function for the system (1.3), therefore, $B_{0}\left(I_{n \mathbf{k}}\right)=0$. The passage of the limit cycle through the resonance zone was described in [18]. If the condition $B_{0} \neq 0,\left|B_{0}\left(I_{n \mathbf{k}}\right)\right|>\max \left|\widetilde{A}\left(v, I_{n \mathbf{k}}\right)\right|$ holds, the averaged system (2.4) does not have equilibria and the resonance level $I=I_{n \mathbf{k}}$ is passable for $\varepsilon$ small enough.

\subsection{Neighborhood of a nonresonance level}

Let us now consider a nonresonance energy level $I=I_{*}$ and make the change of variable $I=I_{*}+\mu W$ in (2.1). As a result, we obtain

$$
\begin{aligned}
\dot{W} & =\mu F_{1}\left(I_{*}, \theta, \theta_{1}, \ldots, \theta_{m}\right)+\mu^{2}\left[\partial F_{1}(\cdot) / \partial I\right] W+O\left(\mu^{3}\right), \\
\dot{\theta} & =\omega\left(I_{*}\right)+\mu b_{1} W+\mu^{2}\left(b_{2} W^{2}+F_{2}\left(I_{*}, \theta, \theta_{1}, \ldots, \theta_{m}\right)\right)+O\left(\mu^{3}\right), \\
\dot{\theta_{k}} & =\omega_{k}, \quad k=1,2, \ldots m,
\end{aligned}
$$

where $b_{1}=\omega^{\prime}\left(I_{*}\right), b_{2}=\frac{1}{2} \omega^{\prime \prime}\left(I_{*}\right)$. Then we make the following change of variable:

$$
u=W+i \mu \sum_{|k| \neq 0} \frac{F_{1 k}\left(I_{*}\right)}{(\bar{k}, \bar{\omega})} e^{i(\bar{k}, \bar{\theta})}+i \mu^{2} \sum_{|k| \neq 0}\left[F_{1 k}^{\prime}\left(I_{*}\right)-b_{1} W k_{0} \frac{F_{1 k}\left(I_{*}\right)}{(\bar{k}, \bar{\omega})}\right] \frac{e^{i(\bar{k}, \bar{\theta})}}{(\bar{k}, \bar{\omega})},
$$

where $F_{1 k}$ are the Fourier expansion coefficients for the function $F_{1}\left(I_{*}, \theta, \theta_{1}, \ldots, \theta_{m}\right), \bar{k}=$ $=\left(k_{0}, k_{1}, \ldots, k_{m}\right), \bar{\theta}=\left(\theta, \theta_{1}, \ldots, \theta_{m}\right), \bar{\omega}=\left(\omega\left(I_{*}\right), \omega_{1}, \ldots, \omega_{m}\right)$. The system takes the form

$$
\begin{aligned}
\dot{u} & =\mu B_{0}\left(I_{*}\right)+\mu^{2} B_{1}\left(I_{*}\right) u+O\left(\mu^{3}\right), \\
\dot{\theta} & =\omega\left(I_{*}\right)+\mu b_{1} u+\mu^{2} Q(u, \theta)+O\left(\mu^{3}\right), \\
\dot{\theta_{k}} & =\omega_{k}, \quad k=1,2, \ldots m,
\end{aligned}
$$

where

$$
\begin{gathered}
B_{0}=F_{10}=\frac{1}{(2 \pi)^{m+1}} \int_{0}^{2 \pi} \ldots \int_{0}^{2 \pi} F_{1}\left(I_{*}, \theta, \theta_{1}, \ldots, \theta_{m}\right) d \theta d \theta_{1} \ldots d \theta_{m}, \\
B_{1}=d B_{0} / d I=\frac{1}{(2 \pi)^{m+1}} \int_{0}^{2 \pi} \ldots \int_{0}^{2 \pi} \frac{\partial F_{1}\left(I_{*}, \theta, \theta_{1}, \ldots, \theta_{m}\right)}{\partial I} d \theta d \theta_{1} \ldots d \theta_{m} .
\end{gathered}
$$


Neglecting terms of order $O\left(\mu^{3}\right)$, we obtain the equation

$$
\dot{u}=\mu B_{0}\left(I_{*}\right)+\mu^{2} B_{1}\left(I_{*}\right) u,
$$

which coincides with the averaged one. Small denominators $(\bar{k}, \bar{\omega})$ may appear in $(2.8)$. This requires an estimate of the frequencies which would provide the series convergence. For example, it will be sufficient if the frequencies satisfy the following condition:

$$
(\bar{k}, \bar{\omega}) \geqslant C /\left(\sum_{j=0}^{m}\left|k_{j}\right|\right)^{m+1},
$$

for all $\left(k_{0}, k_{1}, \ldots, k_{m}\right) \in \mathbf{Z}^{m+1} \backslash\{0\}$. $C$ is a positive constant ${ }^{1}$.

If $B_{0}\left(I_{*}\right) \neq 0$, then the level $I=I_{*}$ is passable. If $B_{0}\left(I_{*}\right)=0$ and $B_{1}\left(I_{*}\right) \neq 0$, then the simple root $u=0$ of (2.10) corresponds to a quasi-periodic solution with the frequencies $\omega\left(I_{*}\right), \omega_{1}, \ldots, \omega_{m}$ in (1.1) (or an $(m+1)$-dimensional invariant torus in (2.1)). Thus, the following theorem holds.

Theorem 2. Assume that the autonomous system (1.3) has a rough limit cycle generated from a nonresonance level $I=I_{*}$ for sufficiently small $\varepsilon>0$ (i.e., $\left.B_{0}\left(I_{*}\right)=0, B_{1}\left(I_{*}\right) \neq 0\right)$ and condition (2.11) is satisfied. Then the system (1.1) has a quasi-periodic solution with the frequencies $\omega\left(I_{*}\right), \omega_{1}, \ldots, \omega_{m}$. The solution corresponds to an $(m+1)$-dimensional invariant torus in the system (2.1), which is asymptotically stable if $B_{1}\left(I_{*}\right)<0$.

\subsection{Global behavior of solutions in $D$}

The averaged system (2.4) is similar to the one obtained in the case of periodic perturbations. It was stated that there is only a finite number of splittable resonances for small $\varepsilon[11]$ (see also [15]). The statement is naturally transferred to the quasi-periodic case. Indeed, replacing scalars by vectors in the appropriate formulas in [11, pp. 188-191], we obtain the following theorem.

Theorem 3. There is only a finite number of resonance levels such that $\max \left|\widetilde{A}\left(v, I_{n \mathbf{k}}\right)\right|>$ $\left|B_{0}\left(I_{n \mathbf{k}}\right)\right|$.

This implies that the system $u^{\prime}=A\left(v ; I_{n \mathbf{k}}\right), \quad v^{\prime}=b_{1} u$ has simple equilibria only for a finite number of resonance levels $I=I_{n \mathbf{k}}$ if $B_{0}\left(I_{n \mathbf{k}}\right) \neq 0$.

Since the system (1.3) has only a finite number of limit cycles by the assumption, there is only a finite number impassable nonresonance levels. This fact and Theorems 1-3 let us establish the global behavior of solutions in $D$. Indeed, the number of the neighborhoods of splittable resonance levels is finite and we can make their $\sqrt{\varepsilon}$-neighborhoods not intersect. Outside these neighborhoods, all the levels $H(x, y)=$ const are passable for $\varepsilon$ small enough. Note that attraction basins of invariant tori can have complex structure due to heteroclinic orbits [11]. If all the levels $H(x, y)=$ const in $D$ are passable, then the qualitative behavior of solutions to (1.1) in $D$ is determined by the autonomous system (1.3).

\section{Splitting of separatrix manifolds}

Without loss of generality, we suppose that the saddle $\left(x_{s}, y_{s}\right)$ of the system (1.1) at $\varepsilon=0$ lies at the origin. $\left(x_{s}, y_{s}\right)$ corresponds to the saddle quasi-periodic solution in the extended phase

${ }^{1}$ This also requires $F_{1}\left(I_{*}, \theta, \theta_{1}, \ldots, \theta_{m}\right)$ to be sufficiently smooth with respect to $\theta, \theta_{1}, \ldots, \theta_{m}$. 
space. Let us denote the coinciding stable and unstable integral manifolds of the unperturbed saddle quasi-periodic solution by $W_{0}^{s}, W_{0}^{u}$. The solution persists under the perturbation when $\varepsilon>0$ small enough and has invariant manifolds $W_{\varepsilon}^{u}, W_{\varepsilon}^{s}$ close to the unperturbed ones [5]. The problem is to analyze the distance between separatrix manifolds $W_{\varepsilon}^{s}$ and $W_{\varepsilon}^{u}$, which coincide in the unperturbed system. For periodic perturbations, one can use the Melnikov formula to determine the distance [4]. In [5], the applicability of the Melnikov formula was extended to sufficiently smooth systems. In [6] the quasi-periodic Melnikov function was derived and a general discussion of the discrete maps construction from the trajectories of time-dependent vector fields was presented. Following [5], we briefly describe the derivation of the Melnikov function in our case.

Let us find the distance $\Delta$ between the manifolds $W_{\varepsilon}^{s}$ and $W_{\varepsilon}^{u}$ of the system (1.1). We assume

$$
f\left(0,0, \omega_{1} t, \ldots, \omega_{m} t\right)=g\left(0,0, \omega_{1} t, \ldots, \omega_{m} t\right)=0
$$

for all $t$. It stands for the manifolds $W_{\varepsilon}^{s, u}(t)$ adjacent to the $t$-axis in the $(x, y, t)$-space.

According to [5], for sufficiently small $\varepsilon$ we have

$$
\begin{gathered}
\sup _{\tau \in[t, \infty)}\left\|W_{0}^{s}(\tau)-W_{\varepsilon}^{s}(\tau)\right\|=O(\varepsilon), \\
\sup _{\tau \in(-\infty, t]}\left\|W_{0}^{u}(\tau)-W_{\varepsilon}^{u}(\tau)\right\|=O(\varepsilon) .
\end{gathered}
$$

Then there exist solutions $x_{\varepsilon}^{s, u}, y_{\varepsilon}^{s, u}$ of (1.1) such that

$$
\lim _{t \rightarrow \infty} x_{\varepsilon}^{s}(t)=\lim _{t \rightarrow-\infty} x_{\varepsilon}^{u}(t)=0, \quad \lim _{t \rightarrow \infty} y_{\varepsilon}^{s}(t)=\lim _{t \rightarrow-\infty} y_{\varepsilon}^{u}(t)=0 .
$$

According to [5], for each $x_{0}^{s, u} y_{0}^{s, u} \subset W_{0}^{s, u}$ we can find $x_{\varepsilon}^{s}, y_{\varepsilon}^{s}$ and $x_{\varepsilon}^{u}, y_{\varepsilon}^{u}$ such that

$$
\begin{aligned}
\sup _{\tau \in[t, \infty]}\left\|x_{0}^{s, u}(\tau)-x_{\varepsilon}^{s}(\tau)\right\|=O\left(\varepsilon\left(1-e^{-C_{1} t}\right)\right), & \sup _{\tau \in[t, \infty]}\left\|y_{0}^{s, u}(\tau)-y_{\varepsilon}^{s}(\tau)\right\|=O\left(\varepsilon\left(1-e^{-C_{1} t}\right)\right), \\
\sup _{\tau \in[-\infty, t]}\left\|x_{0}^{s, u}(\tau)-x_{\varepsilon}^{u}(\tau)\right\|=O\left(\varepsilon\left(1+e^{-C_{1} t}\right)\right), & \sup _{\tau \in[-\infty, t]}\left\|y_{0}^{s, u}(\tau)-y_{\varepsilon}^{u}(\tau)\right\|=O\left(\varepsilon\left(1+e^{-C_{1} t}\right)\right)
\end{aligned}
$$

for certain $C_{1}>0\left(C_{1}\right.$ depends only on the unperturbed system) and for all $t \in R$. Then we define $\xi^{i}, \eta^{i}, i=s, u$ by

$$
\begin{gathered}
x_{\varepsilon}^{i}(t)-x_{0}^{s, u}(t)=\varepsilon \xi^{i}(t, \varepsilon), \\
y_{\varepsilon}^{i}(t)-y_{0}^{s, u}(t)=\varepsilon \eta^{i}(t ; \varepsilon), \\
\left.\left.\sup _{\tau \in[t, \infty]}\left\|\xi^{s}(\tau ; \varepsilon)\right\|=O\left(1+e^{-C_{1} t}\right)\right), \quad \sup _{\tau \in[t, \infty]}\left\|\eta^{s}(\tau ; \varepsilon)\right\|=O\left(1+e^{-C_{1} t}\right)\right), \\
\left.\left.\sup _{\tau \in[-\infty, t]}\left\|\xi^{u}(\tau ; \varepsilon)\right\|=O\left(1+e^{C_{1} t}\right)\right), \sup _{\tau \in[\infty, t]}\left\|\eta^{u}(\tau ; \varepsilon)\right\|=O\left(1+e^{C_{1} t}\right)\right) .
\end{gathered}
$$

Set $x_{0}^{s, u}\left(t_{0}\right)=x_{0}, y_{0}^{s, u}\left(t_{0}\right)=y_{0}$ and define

$$
\Delta_{\varepsilon}^{s, u}\left(t_{0}, x_{0}, y_{0}\right)=\frac{\partial H\left(x_{0}, y_{0}\right)}{\partial y} \eta^{s, u}\left(t_{0} ; \varepsilon\right)+\frac{\partial H\left(x_{0}, y_{0}\right)}{\partial x} \xi^{s, u}\left(t_{0} ; \varepsilon\right),
$$

where $t_{0}$ determines the plane $\left(x, y, t_{0}\right)$ on which we measure the distance. Then the value that determines the distance between the manifolds $W_{\varepsilon}^{s}(t)$ and $W_{\varepsilon}^{u}(t)$ takes the form

$$
\Delta_{\varepsilon}\left(t_{0}, x_{0}, y_{0}\right)=\Delta_{\varepsilon}^{u}\left(t_{0}, x_{0}, y_{0}\right)-\Delta_{\varepsilon}^{s}\left(t_{0}, x_{0}, y_{0}\right) .
$$


Consider the Taylor series at $\varepsilon=0: \Delta_{\varepsilon}\left(t_{0}\right)=\Delta_{0}\left(t_{0}\right)+\varepsilon \Delta_{1}\left(t_{0}\right)+O\left(\varepsilon^{2}\right)=\varepsilon \Delta_{1}\left(t_{0}\right)+O\left(\varepsilon^{2}\right)$. Following [5], we find

$$
\Delta_{1}\left(t_{0}\right)=\int_{-\infty}^{\infty}\left[f\left(x_{0}\left(\tau-t_{0}\right), y_{0}\left(\tau-t_{0}\right), \tau\right) \dot{x_{0}}(\tau-t)-g\left(x_{0}\left(\tau-t_{0}\right), y_{0}\left(\tau-t_{0}\right), \tau\right) \dot{y_{0}}\left(\tau-t_{0}\right)\right] d \tau
$$

$x_{0}(\tau), y_{0}(\tau)$ is the separatrix solution of the unperturbed system, $f \rightarrow f\left(x, y, \omega_{1} \tau+\theta_{10}, \ldots, \omega_{m} \tau+\right.$ $\left.+\theta_{m 0}\right), g \rightarrow g\left(x, y, \omega_{1} \tau+\theta_{10}, \ldots, \omega_{m} \tau+\theta_{m 0}\right), \theta_{10}, \ldots, \theta_{m 0}$ are constants. Since the functions $f, g$ are quasi-periodic in $t$, it follows that $\Delta_{1}\left(t_{0}\right)$ is quasi-periodic as well. Note that $\Delta_{1}$ depends on $\theta_{10}, \ldots, \theta_{m 0}$. This leads to a variety of geometrical interpretations of the stable and unstable manifolds intersections [6].

The fact that $\Delta_{1}\left(t_{0}\right)$ is not sign-preserving implies that $W^{s}\left(t_{0}\right)$ and $W^{u}\left(t_{0}\right)$ intersect transversally and there exist doubly-asymptotic (homoclinic) solutions, i.e, solutions satisfying the condition $\lim _{t \rightarrow \pm \infty} x_{\varepsilon}(t)=\lim _{t \rightarrow \pm \infty} y_{\varepsilon}(t)=0$. The behavior of solutions in the extended neighborhood of a doubly asymptotic solution was studied in [21] and it relates to the existence of a nontrivial hyperbolic set ("irregular" dynamics). In some cases, a stable irregular invariant set of the so-called "quasi-attractor" may appear.

Let us now consider Example (1.4).

\section{Example}

Consider Eq. (1.4)

$$
\left.\ddot{x}+\alpha x+x^{3}=\varepsilon\left[\left(p_{1}-x^{2}\right) \dot{x}+p_{2} \sin t \sin \nu t\right)\right],
$$

$\nu=\sqrt{5}$. If $\alpha=-1$, there are three regions in the phase space of the unperturbed equation filled with closed phase curves. If $\alpha=1$, there exists a unique such region. For simplicity, when we study the global behavior of the solutions to (4.1) in $D$, we take $\alpha=1$ (the behavior in three regions for $\alpha=-1$ can be studied in the same way, e.g., the dissipative case was considered in [14]). For $\alpha=-1$, we will investigate only the neighborhood of the unperturbed separatrix. Note that the equation of type (4.1) for $\alpha=1$ was considered in [18]. However, in [18] only the neighborhoods of resonance levels were studied.

\subsection{Autonomous equation}

The unperturbed equation $(\varepsilon=0)$ admits the following energy integral:

$$
H(x, y)=\dot{x}^{2} / 2+x^{2} / 2+x^{4} / 4=h, h>0
$$

and it has the solution

$$
x(\theta, I)=x_{1} \operatorname{cn}(2 \mathbf{K} \theta / \pi), \theta=\omega t,
$$

where $\omega=\pi(1+4 h)^{1 / 4} /(2 \mathbf{K})$ is a natural frequency, $x_{1}$ is the positive solution of $x^{2} / 2+x^{4} / 4=$ $=h, \operatorname{cn}(u)$ is the Jacobi elliptic function, $\mathbf{K}$ is the complete elliptic integral of the first kind, and $k=k(h)$ is its modulus. Denote the annular region of the phase plane $\{(x, y): H(x, y)=$ $\left.=h, 0<h_{-}<h<h_{+}<\infty\right\}$ by $D$.

The perturbed autonomous system $\left(p_{2}=0\right)$

$$
\dot{x}=y, \dot{y}=-x-x^{3}+\varepsilon\left[\left(p_{1}-x^{2}\right) y\right]
$$


has a unique limit cycle if $p_{1}>0$. It follows from the analysis of the Poincaré-Pontryagin generating function, which has the following form (up to the factor $4 /\left(3 \pi(1-2 \rho)^{5 / 2}\right)$ ):

$$
\begin{array}{r}
B_{0}(\rho)=p_{1}\left[(1-\rho)(1-2 \rho) \mathbf{K}-(1-2 \rho)^{2} \mathbf{E}\right]- \\
-\frac{2}{5}\left[(\rho-1)(2-\rho) \mathbf{K}+2\left(\rho^{2}-\rho+1\right) \mathbf{E}\right],
\end{array}
$$

where $\mathbf{E}$ is the complete elliptic integral of the second kind, $\rho=k^{2}=\frac{-1+\sqrt{1+4 h}}{2 \sqrt{1+4 h}}$.

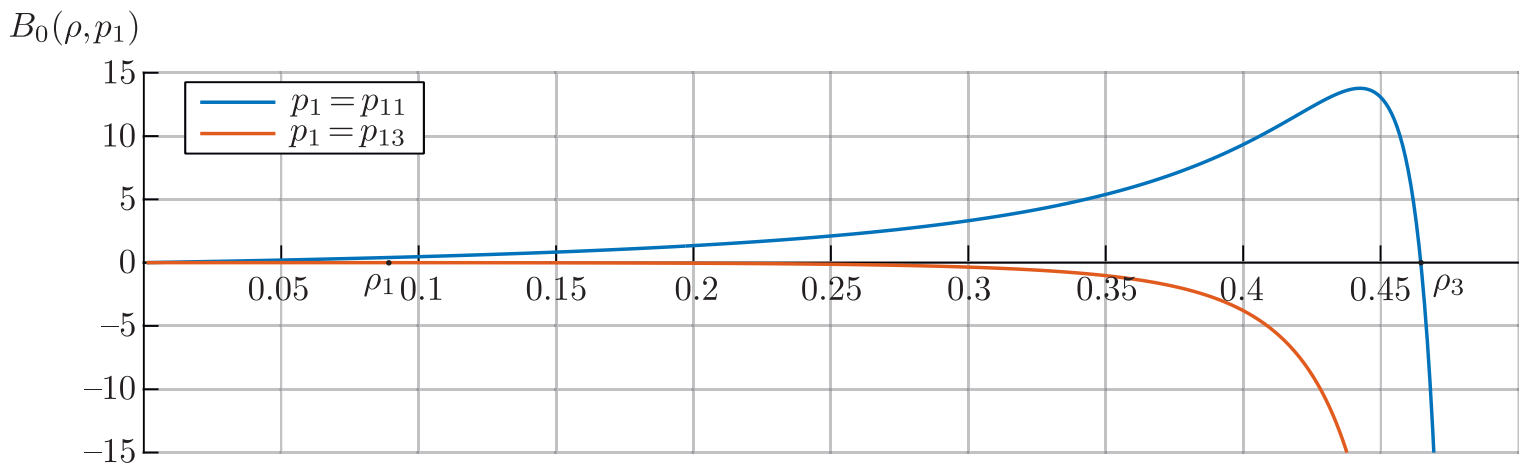

Fig. 1. $B_{0}\left(\rho ; p_{13}\right)$ and $B_{0}\left(\rho ; p_{11}\right)$.

Let $\rho_{*} \in(0,1 / 2)$ be a simple zero of $B_{0}(\rho)$. One can see that $p_{1} \rightarrow \infty$ as $\rho_{*} \rightarrow 0$ and $p_{1} \rightarrow+0$ as $\rho_{*} \rightarrow 1 / 2$. It is well known that $\rho_{*}$ determines the level of the unperturbed system which generates a rough limit cycle in (4.4). If $B^{\prime}\left(\rho_{*}\right)<0$ and $\varepsilon>0$, the cycle is stable. The sign of $B^{\prime}\left(\rho_{*}\right)$ coincides with the sign of $\sigma\left(\rho_{*}\right)$, where

$$
\sigma(\rho)=p_{1}-\frac{2}{(1-2 \rho) \mathbf{K}}[\mathbf{E}-(1-\rho) \mathbf{K}] .
$$

Figure 1 shows the function $B_{0}\left(\rho ; p_{1}\right)$ for two values of $p_{1}$, where $p_{31}$ is found from the condition $B_{0}\left(\rho\left(h_{3 \mathbf{k}}\right) ; p\right)=0$ and $p_{11}$ is found from the condition $B_{0}\left(\rho\left(h_{1 \mathbf{k}}\right) ; p\right)=0$. Simple eqiulibria of the equation $B_{0}\left(\rho ; p_{1}\right)=0$ correspond to rough limit cycles in (4.4) [15].

\subsection{Nonautonomous equation}

The behavior of solutions in the neighborhoods of individual resonance levels is described by the averaged system (2.4), where

$$
\begin{gathered}
A(v)=B+\frac{p_{2}}{4 \pi^{2} n^{2}} \int_{0}^{2 \pi n} \int_{0}^{2 \pi n} \sin \theta_{1} \sin \theta_{2} x_{\theta}^{\prime}\left(v+\left(k_{1} \theta_{1}+k_{2} \theta_{2}\right) / n\right) d \theta_{1} d \theta_{2}= \\
=\left\{\begin{array}{c}
B+p_{2} \sqrt{2}\left(k_{1} \omega_{1}+k_{2} \omega_{2}\right) \frac{a^{n / 2}}{1+a^{n}} \sin n v \\
\text { if } n \text { is odd, } k_{1}= \pm 1, k_{2}=1, \\
B_{0} \quad \text { if } n \text { is even or } k_{1} \neq \pm 1, k_{2} \neq 1,
\end{array}\right.
\end{gathered}
$$

$\sigma\left(I_{n \mathbf{k}}\right)$ is determined by formula (4.6). 


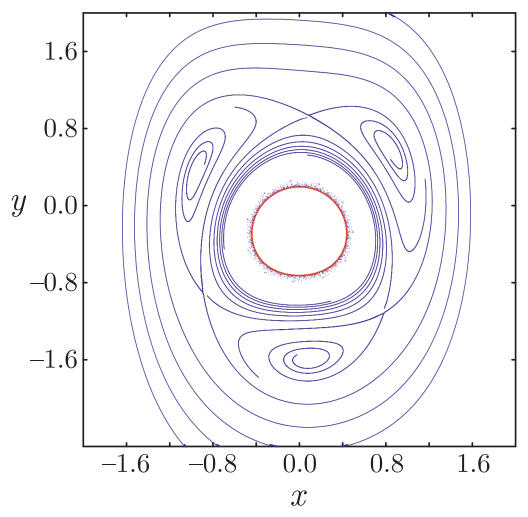

(a)

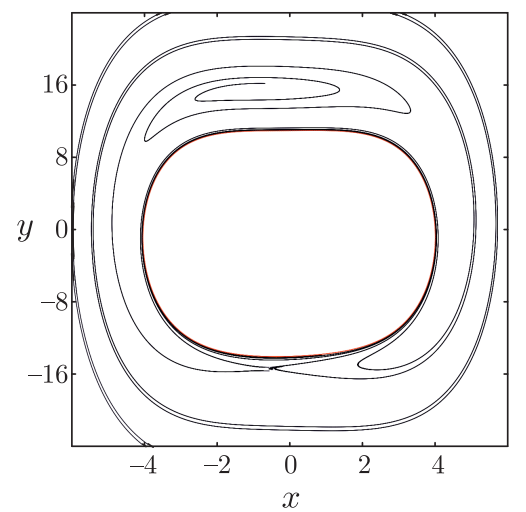

(d)

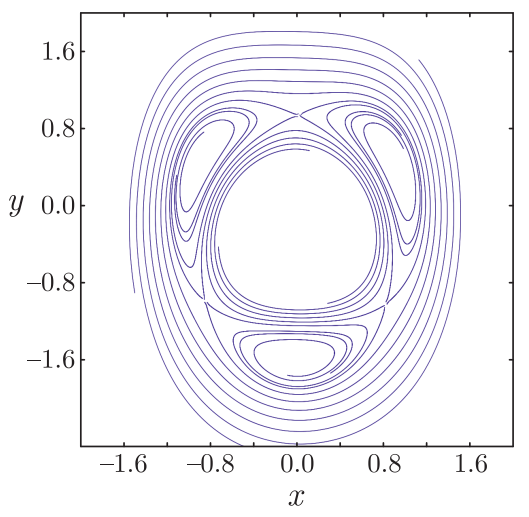

(b)

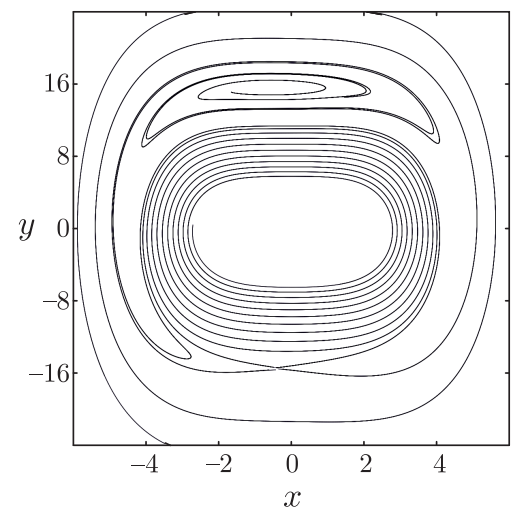

(e)

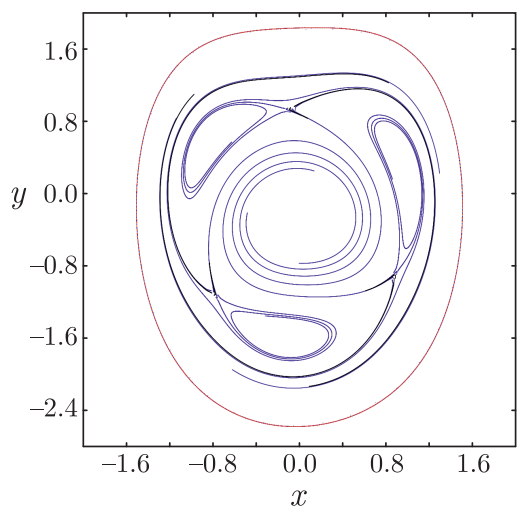

(c)

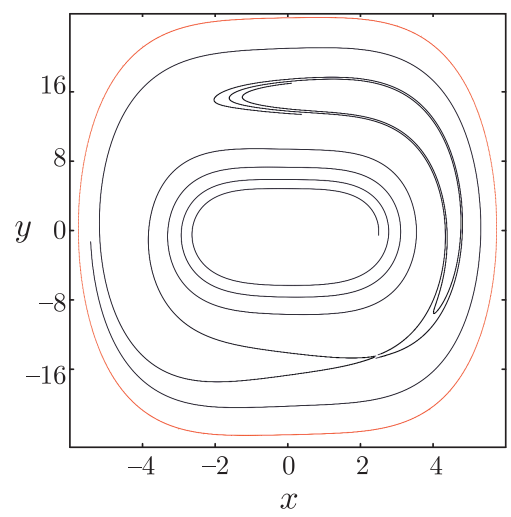

(f)

Fig. 2. Phase portraits of (2.4) in the $(x, y)$-plane in the neighborhood of $H(x, y)=h_{311}$ : (a), (b), (c) and $H(x, y)=h_{111}:(\mathrm{d}),(\mathrm{e}),(\mathrm{f})$.

From the conditions $\omega=( \pm 1+\sqrt{5}) / n$ and $\omega>1$, one can find that only three "splittable" resonance levels $H(x, y)=h_{311}, H(x, y)=h_{1,-1,1}, H(x, y)=h_{111}\left(h_{311}<h_{1,-1,1}<h_{111}\right)$ are possible.

Thus, we have $u^{\prime}=B_{0}\left(I_{n \mathbf{k}}\right)+\mu \sigma u$ for almost all resonance levels. If $B_{0} \neq 0$, then the level $I=I_{n \mathbf{k}}$ is passable. If $B_{0}=0$ and $\sigma<0$ (the autonomous system (1.3) has a stable limit cycle in the neighborhood of $I=I_{n \mathbf{k}}$ ), then $u=0$ is a stable equilibrium. This implies that there exists a stable invariant curve embracing the phase cylinder of the averaged system (2.4) and a 3 -dimensional stable torus in the system (2.1) (see Theorem 2). By varying $p_{1}$, one can see the passage of this torus through resonance. Figure 2 shows phase portraits of the averaged system for resonances with $n=3$ and $n=1$. There is a stable limit cycle of the averaged system for $\varepsilon=0.1, p_{1}=0.005, n=3$ (Fig. 2a). The cycle corresponds to a 3 -dimensional torus in the initial system (2.1). As $p_{1}$ increases, the cycle comes into the neighborhood of the resonance level $I=I_{311}\left(H(x, y)=h_{311}\right)$. The passage through a resonance zone is described in [18]. In Fig. $2 b$, there are no cycles and the oscillations are synchronized at the frequencies $\omega_{1} / 3, \omega_{2} / 3$. Then, as $p_{1}$ increases further, a cycle outside the resonance occurs (Fig. 2c, where $p_{1}=0.06$ ). The cycle is growing along with $p_{1}$ and is getting closer to the resonance level $H(x, y)=h_{1,-1,1}$. Further, the cycle passes through this zone and, finally, the resonance zone with $H(x, y)=h_{111}$ (see Figs. 2d-2f). The dynamics of the averaged system in the neighborhood of $H(x, y)=h_{1,-1,1}$ is similar to the one in the neighborhood of $H(x, y)=h_{111}$. 


\subsection{The neighborhood of the unperturbed separatrix}

We now consider the equation

$$
\ddot{x}-x+x^{3}=\varepsilon\left[\left(p_{1}-x^{2}\right) y+p_{2} \sin t \sin \nu t\right] .
$$

The saddle of the unperturbed system lies at the origin. To fix the saddle solution, we make a change of variable $x=\xi+\varepsilon x_{1}(t)+O\left(\varepsilon^{2}\right)$. Then, neglecting terms of order $O\left(\varepsilon^{2}\right)$ ), we obtain the following equation:

$$
\ddot{\xi}-\xi+\xi^{3}=\varepsilon\left[\left(p_{1}-\xi^{2}\right) \dot{\xi}-3 \xi^{2} x_{1}(t)\right],
$$

where

$$
x_{1}(t)=\frac{p_{2}}{2}\left[\frac{\cos (1-\nu) t}{1+(1-\nu)^{2}}+\frac{\cos (1+\nu) t}{1+(1+\nu)^{2}}\right] .
$$

It follows from (3.3) that

$$
\Delta_{\varepsilon}\left(t_{0}\right)=\varepsilon \Delta_{0}\left(t_{0}\right)+O\left(\varepsilon^{2}\right),
$$

where

$$
\Delta_{0}\left(t_{0}\right)=\int_{\infty}^{\infty}\left[\left(p_{1}-\xi_{s}^{2}\left(t-t_{0}\right)\right) \dot{\xi}_{s}\left(t-t_{0}\right)-3 \xi_{s}^{2}\left(t-t_{0}\right) x_{1}(t)\right] \dot{\xi}_{s}\left(t-t_{0}\right) d t
$$

Here

$$
\xi_{s}(t)= \pm \sqrt{2} / \cosh t, \quad \dot{\xi}_{s}(t)=\mp \sqrt{2} \sinh (t) / \operatorname{ch}^{2}(t)
$$

is the separatrix solution to the unperturbed equation. Here the sign "+" corresponds to the right separatrix loop and the sign "-" corresponds to the left separatrix loop.

The result is [20]

$$
\Delta_{0}\left(t_{0}\right)=5 p_{1}-4+p_{2} \frac{\pi \sqrt{2}}{2}\left[B_{1} \sin (1+\nu) t_{0}+B_{2} \sin (1-\nu) t_{0}\right],
$$

where

$$
B_{1,2}=\frac{(1 \pm \nu)}{\cosh ((1 \pm \nu) \pi / 2)}
$$

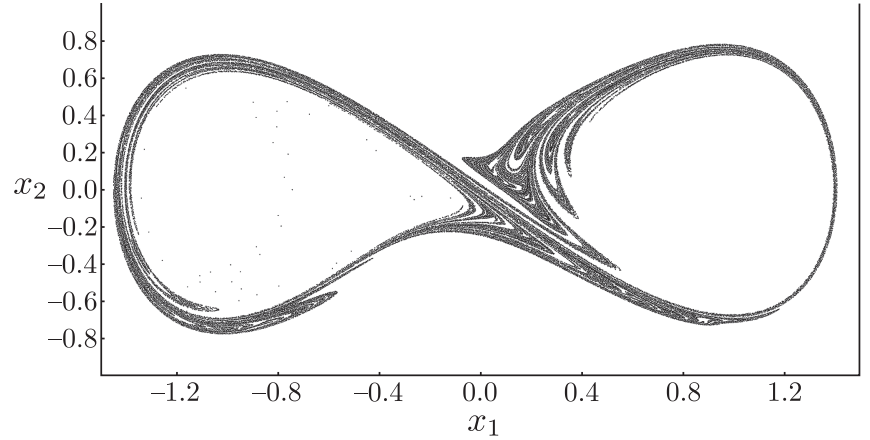

(a)

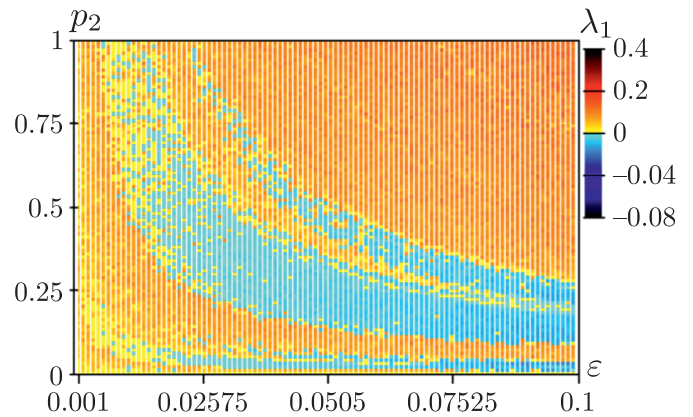

(b)

Fig. 3. Irregular repelling set (a) and chart of the senior Lyapunov exponent (b).

Note that the Melnikov function is quasi-periodic, as is the perturbation. From (4.10) one can conclude that if $\left|p_{1}\right|<(3 / 8) \sqrt{2} \pi\left|p_{2}\right| \sqrt{B_{1}^{2}+B_{2}^{2}}$ is satisfied, then the function $\Delta_{0}\left(t_{0}\right)$ is signalternating and, therefore, $W_{\varepsilon}^{s} \cap W_{\varepsilon}^{u} \neq \emptyset$. This implies that there exist homoclinic solutions 
to the saddle solution. They correspond to the homoclinic orbits of the Poincare map. The structure of the neighborhood of these orbits was studied in [21].

Note that the unstable limit cycle of the autonomous perturbed system $\left(p_{2}=0\right)$ merges with the separatrix and forms an unstable separatrix loop if $p_{1}=4 / 5$. In this case, the free term of $\Delta_{0}$ is neglected and a irregular repelling set ${ }^{2}$ can appear. Such a set is shown in Fig. 3a for $\varepsilon=0.1, p_{1}=0.8, p_{2}=0.8$ (the fractional part of the Lyapunov dimension equals $\approx 0.826$ ). Figure $3 \mathrm{~b}$ shows a chart of the largest Lyapunov exponent $\lambda_{1}$ in the $\left(\varepsilon, p_{2}\right)$-plane for $p_{1}=0.8$. There are regions with $\lambda_{1}<0$ that correspond to regular asymptotic behavior $(t \rightarrow-\infty)$ in the neighborhood of the unperturbed separatrix (unstable quasi-periodic solutions).

\section{References}

[1] Jing, Zh., Huang, J., and Deng, J., Complex Dynamics in Three-Well Duffing System with Two External Forcings, Chaos Solitons Fractals, 2007, vol.33, no. 3, pp. 795-812.

[2] Liu, B. and You, J., Quasiperiodic Solutions of Duffing's Equations, Nonlinear Anal., 1998, vol. 33, no. 6, pp. 645-655.

[3] Jing, Zh., Yang, Zh., and Jiang, T., Complex Dynamics in Duffing-van der Pol Equation, Chaos Solitons Fractals, 2006, vol.27, no.3, pp. 722-747.

[4] Mel'nikov, V. K., On the Stability of a Center for Time-Periodic Perturbations, Tr. Mosk. Mat. Obs., 1963, vol. 12, pp. 3-52 (Russian).

[5] Sanders, J. A., Melnikov's Method and Averaging, Celestial Mech., 1982, vol.28, nos. 1-2, pp. 171181.

[6] Wiggins, S., Chaotic Transport in Dynamical Systems, Interdiscip. Appl. Math., vol.2, New York: Springer, 1992.

[7] Grischenko, A.D. and Vavriv, D. M., Dynamics of Pendulum with a Quasiperiodic Perturbation, Tech. Phys., 1997, vol.42, no.10, pp.1115-1120; see also: Zh. Tekh. Fiz., 1997, vol.67, no.10, pp. 1-7.

[8] Yagasaki, K., Second-Order Averaging and Chaos in Quasiperiodically Forced Weakly Nonlinear Oscillators, Phys. D, 1990, vol.44, no. 3, pp. 445-458.

[9] Belogortsev, A. B., Quasiperiodic Resonance and Bifurcations of Tori in the Weakly Nonlinear Duffing Oscillator, Phys. D, 1992, vol. 59, no. 4, pp. 417-429.

[10] Jing, Zh. and Wang, R., Complex Dynamics in Duffing System with Two External Forcings, Chaos Solitons Fractals, 2005, vol. 23, no. 2, pp. 399-411.

[11] Morozov, A. D., Quasi-Conservative Systems: Cycles, Resonances and Chaos, World Sci. Ser. Nonlinear Sci. Ser. A Monogr. Treatises, vol. 30, River Edge, N.J.: World Sci., 1999.

[12] Bogoliubov, N. N. and Mitropolsky, Yu. A., Asymptotic Methods in the Theory of Non-Linear Oscillations, New York: Gordon \& Breach, 1961.

[13] Mitropolsky, Yu. A. and Lykova, O. B., Integrated Manifolds in the Nonlinear Mechanics, Moscow: Nauka, 1973 (Russian).

[14] Morozov, A. D. and Morozov, K.E., Quasiperiodic Perturbations of Two-Dimensional Hamiltonian Systems, Differ. Equ., 2017, vol.53, no.12, pp.1557-1566; see also: Differ. Uravn., 2017, vol. 53, no. 12, pp. 1607-1615.

[15] Morozov, A.D., Resonances, Cycles and Chaos in Quasi-Conservative Systems, Moscow-Izhevsk: R\&C Dynamics, Institute of Computer Science, 2005 (Russian).

[16] Morozov, A. D., On the Structure of Resonance Zones and Chaos in Nonlinear Parametric Systems, Internat. J. Bifur. Chaos Appl. Sci. Engrg., 1994, vol. 4, no. 2, pp. 401-410.

\footnotetext{
${ }^{2}$ One can make this set stable (the so-called quasi-attractor [15]) by the change $t \rightarrow-t$.
} 
[17] Morozov, A. D., Resonances and Chaos in Parametric Systems, J. Appl. Math. Mech., 1994, vol. 58, no. 3, pp.413-423; see also: Prikl. Mat. Mekh., 1994, vol. 58, no. 3, pp.41-51.

[18] Morozov, A.D. and Morozov, K.E., On Synchronization of Quasiperiodic Oscillations, Russian J. Nonlinear Dyn., 2018, vol. 14, no. 3, pp. 367-376.

[19] Hale, J. K., Oscillations in Nonlinear System, New York: McGraw-Hill, 1963.

[20] Morozov, A.D. and Dragunov,T.N. On Quasi-periodic Perturbations of Duffing Equation, Interdiscip. J. Discontin. Nonlinearity Complex, 2016, vol.5, no.4, pp. 377-386.

[21] Shilnikov, L. P., On a Poincaré-Birkhoff Problem, Math. USSR-Sb., 1967, vol. 3, no. 3, pp. 353-371; see also: Mat. Sb. (N.S.), 1967, vol.74(116), no. 3, pp. 378-397. 